\title{
NOTE ON ABSOLUTELY CONVERGENT DIRICHLET SERIES
}

\author{
EDWIN HEWITT ${ }^{1}$ AND J. H. WILLIAMSON ${ }^{2}$
}

In view of Wiener's well-known theorem [11, p. 91], on the inverses of absolutely convergent Fourier series, it is natural to ask whether something similar is true for Dirichlet series. If $s=\sigma+i t$ is a complex variable and $f(s)$ is representable for $\sigma \geqq 0$ as

$$
\sum_{n=1}^{\infty} a_{n} n^{-s}, \text { where } \sum_{n=1}^{\infty}\left|a_{n}\right|<\infty,
$$

is it true that $|f(s)| \geqq k>0$ for $\sigma \geqq 0$ implies that $(f(s))^{-1}$ is also of the form (1)? In this note, an affirmative answer is supplied. ${ }^{3}$

Let $P$ be the semigroup of positive integers under multiplication, and let $l_{1}(P)$ be the class of all complex functions $a$ on $P, a=\left\{a_{n}\right\}_{n=1}^{\infty}$, for which $\|a\|=\sum_{n=1}^{\infty}\left|a_{n}\right|$ is finite. We obtain a commutative Banach algebra by defining $(\alpha a)_{n}=\alpha a_{n}$ for complex $\alpha,(a+b)_{n}=a_{n}+b_{n}$, and the product $a * b$ (convolution) by $(a * b)_{n}=\sum_{j k=n} a_{j} b_{k}$. The algebra $l_{1}(P)$ has as its unit $e$ the function such that $e_{1}=1$ and $e_{n}=0$ if $n>1$. The theory of $l_{1}$-algebras of commutative semigroups is developed in [4] and [5]. The present note is based on the observation that the Dirichlet series (1) is the Fourier transform of the element $a \in l_{1}(P)$, defined on a very small subspace of the space of all maximal ideals of $l_{1}(P)$.

Lemma 1. Let $a \in l_{1}(P)$. Then $a^{-1}$ exists if and only if

$$
\hat{a}(\chi)=\sum_{n=1}^{\infty} a_{n} \chi(n)
$$

is different from zero for all bounded complex functions $\chi$ on $P$, not identically zero, such that $\chi(m n)=\chi(m) \chi(n)$ identically ("semicharacters" of $P$ ).

PROOF. This follows immediately from Theorem 2.7 of [5], where it is shown that the multiplicative linear functionals on $l_{1}(P)$ are

Received by the editors September 4, 1956 and, in revised form, January 10, 1957.

${ }^{1}$ Fellow of the John Simon Guggenheim Memorial Foundation, and also supported in part by the National Science Foundation.

2 Commonwealth Fund Fellow.

${ }^{3}$ In $[1,6$, and 7$]$, the problem of inversion of nonabsolutely convergent Dirichlet series is studied. The results of these papers seem to have little to do with those of the present note. 
exactly the mappings $a \rightarrow \hat{a}(\chi)$ defined in (2). Theorem 5.8 of [5] shows that $l_{1}(P)$ is semisimple.

We next compute the semicharacters of $P$. If $\chi$ is a semicharacter of $P$, then $\chi(1)=1$, and $\chi(p)$ can be any complex number such that $0 \leqq|\chi(p)| \leqq 1$, for each prime $p \cdot{ }^{4}$ Then for $n=\prod p_{r}^{\beta_{r}}$, we have $\chi(n)$ $=\prod \chi\left(p_{r}\right)^{\beta_{r}}$. The set $\hat{P}$ of all semicharacters of $P$ is obviously a semigroup under pointwise multiplication, isomorphic to the Cartesian product of a countably infinite number of closed unit disks $\{z:|z| \leqq 1\}$. The Gel'fand topology for $\hat{P}$ (the weakest topology for $\hat{P}$ under which all functions $\hat{a}$ as in (2) are continuous, for $a \in l_{1}(P)$ ), is easily shown to be the usual Cartesian product topology. The very special semicharacters $\chi_{s}$, where $\chi_{s}(n)=n^{-s}(\sigma \geqq 0)$, give us the functions

$$
f_{a}(s)=\hat{a}\left(\chi_{s}\right)=\sum_{n=1}^{\infty} a_{n} n^{-s} .
$$

The uniqueness theorem for Dirichlet series shows again that $l_{1}(P)$ is semisimple. It is easy to see that the set $\left\{\chi_{s}\right\}_{\sigma \geq 0}$ is not dense in $\hat{P}$. For example, if $\psi$ is the semicharacter such that $\psi(2)=0, \psi(3)=1$, and $\psi(p)=0$ for all primes $>3$, then $\psi$ is not in the closure of $\left\{\chi_{s}\right\}_{\sigma \geq 0}$.

Gel'fand's famous proof [2] of Wiener's theorem on absolutely convergent Fourier series amounts simply to noting that the mappings $\left\{a_{n}\right\}_{n=-\infty}^{+\infty} \rightarrow \sum_{n=-\infty}^{+\infty} a_{n} e^{i n \theta}(-\pi \leqq \theta<\pi)$ are the only multiplicative linear functionals on the $l_{1}$-algebra of the additive group of all integers. Our present problem is complicated by the fact that the mappings $a \rightarrow \hat{a}\left(\chi_{s}\right)$ defined in (3) are not even dense in the space of all multiplicative linear functionals on $l_{1}(P)$. To get around this difficulty, we need more refined properties of $\hat{P}$ and the functions $a$. Let $z=\left(z_{1}, \cdots, z_{N}\right)$ be a point in complex $N$-space, and let $\phi(z)$ be any polynomial in $z_{1}, \cdots, z_{N}$. Let $q_{1}, \cdots, q_{N}$ be any real numbers all greater than 1 . For $\sigma \geqq 0$, let $D_{\sigma}=\left\{z:\left|z_{i}\right| \leqq q_{i}^{-\sigma}, i=1, \cdots, N\right\}$. In the principal application, $q_{1}, \cdots, q_{N}$ will be the first $N$ primes. Let $\epsilon$ be any positive real number.

Lemma 2. If $|\phi(z)|<\epsilon$ for some $z \in D_{0}$, then there is a number $\sigma \geqq 0$ and a point $z^{\prime}$ with $\left|z_{i}^{\prime}\right|=q_{i}^{-\sigma}(i=1, \cdots, N)$ such that $\left|\phi\left(z^{\prime}\right)\right|<\epsilon$.

Proof. It is evident that the set $\phi\left(D_{\sigma}\right)$ varies continuously with $\sigma$. As $\sigma \rightarrow+\infty, \phi\left(D_{\sigma}\right)$ shrinks to $\phi(0)$. If $\phi(0)=0$, we can clearly make $\left|\phi\left(z^{\prime}\right)\right|<\epsilon$ by taking $z_{i}^{\prime}=q_{i}^{-\sigma}(i=1, \cdots, N)$ for sufficiently large $\sigma$.

If $\phi(0) \neq 0$, then, since $|\phi(z)|<\epsilon$ for some $z \in D_{0}$ and $0 \notin \phi\left(D_{\sigma}\right)$ for sufficiently large $\sigma$, we can find a $\sigma$ such that $0 \notin \phi\left(D_{\sigma}\right)$ and $|\phi(z)|<\epsilon$ for some $z \in D_{\sigma}$. For instance, writing $\eta=\min [\epsilon / 2,|\phi(0)| / 2]$, take

4 We begin the series of primes with 2 . 
$\sigma=\inf \tau$, over all $\tau$ such that $|\phi(z)| \geqq \eta$ for all $z \in D_{r}$. Let $\sigma$ be any such value, and fixed.

Since $D_{\sigma}$ is compact, the infimum $\inf _{z \in D_{\sigma}}|\phi(z)|$ is attained: say for $\boldsymbol{z}^{\prime}=\left(z_{1}^{\prime}, \cdots, z_{N}^{\prime}\right)$. Let $\theta\left(z_{r}\right)=\phi\left(z_{1}^{\prime}, \cdots, z_{r}, \cdots, z_{N}^{\prime}\right)(r=1, \cdots, N)$. Since $\theta\left(z_{r}\right) \neq 0$ for $\left|z_{r}\right| \leqq q_{r}^{-\sigma}$, it follows that $\inf _{\left|z_{r}\right| \leq q_{r}-\sigma}\left|\theta\left(z_{r}\right)\right|$ $=\inf _{z \in D_{e}}|\phi(z)|$ is attained only for $\left|z_{r}\right|=q_{r}^{-\sigma}$, by applying the maximum modulus principle to $\left(\theta\left(\boldsymbol{z}_{r}\right)\right)^{-1}$. That is, $\left|\boldsymbol{z}_{r}^{\prime}\right|=q_{r}^{-\sigma}$. Since this is true for all $r, r=1, \cdots, N$, the result holds as stated.

CoRollary. If $\phi(z)=0$ for some $z \in D_{0}$, then there is a value of $\sigma$, and a point $\boldsymbol{z}^{\prime}=\left(z_{1}^{\prime}, \cdots, z_{N}^{\prime}\right)$ with $\left|z_{i}^{\prime}\right|=q_{i^{-\sigma}}(i=1, \cdots, N)$ such that $\phi\left(z^{\prime}\right)=0$.

This follows from Lemma 2 by a compactness argument.

Next, let $S=\left\{q_{1}, q_{2}, \cdots\right\}$ be any sequence of real numbers, all greater than 1 , such that the set $\left\{\log q_{1}, \log q_{2}, \ldots\right\}$ is linearly independent over the rational number field. For $\sigma \geqq 0$, let $M_{\sigma}=M_{\sigma}(S)$ be the set of all semicharacters $\chi \in \hat{P}$ such that $\left|\chi\left(p_{i}\right)\right|=q_{i}^{-\sigma}$ for each prime $p_{i}$, and write $M_{\infty}$ for the set whose sole element is the semicharacter $\chi$ such that $\chi(p)=0$ for all primes $p$. For $\sigma \geqq 0$, let $L_{\sigma}$ $=L_{\sigma}(S)$ be the set of all semicharacters $\chi$ such that $\chi\left(p_{j}\right)=q_{j}^{-\sigma-i t}$ for each prime $p_{j}$, where $t$ is a real number that depends upon $\chi$ but not upon $j$.

Lemma 3. For each $\sigma \geqq 0$, we have $\bar{L}_{\sigma}=M_{\text {c }}$ (- denotes closure in the Gel'fand topology for $\hat{P}) .^{5}$

Proof. Clearly $M_{\sigma} \supset L_{\sigma}$, and $M_{\sigma}$ is closed, so we have only to prove that $\bar{L}_{\sigma} \supset M_{\sigma}$. To simplify the notation, take $\sigma=0$ : the proof for $\sigma>0$ will follow at once. We have to prove that, given $\chi \in M_{0}$, a positive integer $N$, and $\delta>0$, there is a real number $t$ such that

$$
\left|\chi\left(p_{r}\right)-q_{r}^{-i t}\right|<\delta \quad(r=1, \cdots, N),
$$

where $p_{1}, \cdots, p_{N}$ are the first $N$ primes. Writing $\chi\left(p_{r}\right)=e^{-i \psi(r)}$, where $\psi(r)$ is real, we rewrite (4) as

$$
\left|e^{-i \psi(r)}-e^{-i t \log q_{r}}\right|<\delta \quad(r=1, \cdots, N) .
$$

Since the numbers $\log q_{r}$ are by hypothesis linearly independent, the Kronecker approximation theorem (Theorem 444 of [3]) can be applied. A simple computation, which we omit, shows that (5) can be satisfied for a real number $t$.

We now give our main result.

THEOREM 1. The following assertions are equivalent.

- This lemma is closely related to Satz I of [1]. 
1. The element $a \in l_{1}(P)$ has an inverse $a^{-1}$ in $l_{1}(P)$.

2. The function $f_{a}$, where $f_{a}(s)=\sum_{n=1}^{\infty} a_{n} n^{-s}$, is bounded away from zero in absolute value for $\sigma \geqq 0$.

3. $f_{a}(s)$ vanishes for no $s$ with $\sigma \geqq 0$, and $\left(f_{a}(s)\right)^{-1}=\sum_{n=1}^{\infty} b_{n} n^{-8}$ for $\sigma \geqq 0$, where $\sum_{n=1}^{\infty}\left|b_{n}\right|$ is finite.

Proof. The equivalence of 1 . and 3 . follows at once from the uniqueness theorem for Dirichlet series. It is obvious that 3 . implies 2. We have then only to prove that 2 . implies 1 . Suppose that $a \in l_{1}(P)$ has no inverse in $l_{1}(P)$. By Lemma 1 , there is a semicharacter $\chi \in \hat{P}$ such that (2) is zero for this choice of $\chi$. Given $\epsilon>0$, choose the positive integer $M$ so that $\sum_{n=M+1}^{\infty}\left|a_{n}\right|<\epsilon$ : it then follows that $\left|\sum_{n=1}^{M} a_{n} \chi(n)\right|<\epsilon$. Now, $\sum_{n=1}^{M} a_{n} \chi(n)$ is a polynomial in $\chi(2)$, $\chi(3), \cdots, \chi\left(p_{N}\right)$, where $p_{N}$ is the largest prime not exceeding $M$. Apply Lemma 2 with $q_{i}=p_{i}\left(p_{i}\right.$ is the $i$ th prime): there is a $\sigma \geqq 0$ and a semicharacter $\psi \in M_{\sigma}$ such that $\left|\sum_{n=1}^{M} a_{n} \psi(n)\right|<\epsilon$. We obviously have $\left|\sum_{n=1}^{\infty} a_{n} \psi(n)\right|<2 \epsilon$. Now use Lemma 3 , again with $q_{i}=p_{i}$. It is immediate that there is a real number $t$ such that $\left|\sum_{n=1}^{\infty} a_{n} n^{-\sigma-i t}\right|<3 \epsilon$. Since $\epsilon$ is an arbitrary positive number, the function $f_{a}(s)$ is not bounded away from zero in absolute value for $\sigma \geqq 0$. This completes the proof.

We make now some additional comments, and point out some related results.

The case of Dirichlet series with abscissa of absolute convergence different from zero can easily be reduced to the case just treated. If the series $\sum_{n=1}^{\infty} a_{n} n^{-s}$ converges absolutely for $\sigma \geqq \beta$, then the function $b=\left\{a_{n} n^{-\beta}\right\}_{n=1}^{\infty}$, defined on $P$, is in the algebra $l_{1}(P)$. A theorem like Theorem 1 follows at once.

The following situation is simpler than that of Theorem 1. Let $Q$ denote the multiplicative group of positive rational numbers, and $l_{1}(Q)$ the class of functions $a=\left\{a_{r}\right\}_{r \in Q}$ such that $\sum_{r \in Q}\left|a_{r}\right|$ is finite. This function class can be treated as the $\Omega_{1}$-algebra of the discrete commutative group $Q$. Kronecker's theorem implies, just as in Lemma 3, that the characters $\chi(r)=e^{-i \ell \log r}(r \in Q)$ are dense in the dual group $\hat{Q}$, and hence the analogue of Theorem 1 holds for "Dirichlet" series $\sum_{r \in Q} a_{r} r^{-i t}$, i.e.

THEOREM 2. A necessary and sufficient condition that $a \in l_{1}(Q)$ should have an inverse in $l_{1}(Q)$ is that the function $\left|\sum_{r \in Q} a_{r} r^{-i t}\right|$ should be bounded away from zero for all real $t$. (Since commutative group algebras are regular in the sense of Silov (see for example [8, p. 57 and p. 146]), we can assert the following: Let $B$ be any subset of $\hat{Q}$; then every function $a \in l_{1}(Q)$ such that $|\hat{a}|$ is bounded away from zero on $B$ has an inverse in $l_{1}(Q)$ if and only if $B$ is dense in $\hat{Q}$.) 
Let $M_{\sigma}=M_{\sigma}(S)$ be defined as above for any sequence of real numbers $S=\left\{q_{1}, q_{2}, \cdots\right\}$ all greater than 1 , irrespective of whether $\left\{\log q_{1}, \log q_{2}, \cdots\right\}$ is a linearly independent set. Let $M_{0}$ be the set of all $\chi \in \hat{P}$ such that $|\chi(n)|=1$ for all $n \in P$. Let $M(S)=\bigcup_{0 \leq 0<\infty} M_{\sigma}(S)$. Then we have

Theorem 3. $M(S)$ is a proper closed subset of the space $\hat{P}$. For each $a \in l_{1}(P)$, the set of values $\{a(\chi): \chi \in M(S)\}$ is the same as the set of values $\{a(\chi): \chi \in \hat{P}\}$. If, for some $\lambda \geqq 0, q_{i}^{\prime}=q_{i}^{\lambda}(1 \leqq i<\infty)$, then $M(S)=M\left(S^{\prime}\right)$; otherwise $M(S) \cap M\left(S^{\prime}\right)=M_{0} \cup M_{\infty}$. The set $M_{0}$ is the Silov boundary of $\hat{P}$.

Proof. It is an easy deduction from Lemma 2 that if $a(\chi)=0$ for some $\chi \in \hat{P}$, then $\hat{a}(\chi)=0$ for some $\chi \in M(S)$. The first statement follows from this upon looking at $a-\alpha e$ instead of $a$. We omit the other details.

Suppose now that $\left\{\log q_{1}, \log q_{2}, \cdots\right\}$ is a rationally independent set of numbers, and write $L(S)=\bigcup_{0 \leqq \sigma<\infty} L_{\sigma}(S)$. Then it is immediate, from Lemma 3, that $\bar{L}(S)=M(S)$. For $n=\prod p_{r}^{\beta_{r}}$, let $h_{n}=\prod q_{r}^{\beta_{r}}$. If we write $f_{a}(S, s)=\sum_{n=1}^{\infty} a_{n} h_{n}^{-s}$, we can clearly assert

TheOREm 4. A necessary and sufficient condition that $a \in l_{1}(P)$ should have an inverse in $l_{1}(P)$ is that $\left|f_{a}(S, s)\right|$ should be bounded away from zero for $\sigma \geqq 0$.

Another immediate consequence is

THEOREM 5. The closure of the set of values of $f_{a}(S, s)$ for $\sigma \geqq 0$ is the same as that of $f_{a}\left(S_{0}, s\right)$ for $\sigma \geqq 0$, whenever $S$ and $S_{0}$ are sets of numbers $>1$ each with rationally independent logarithms.

The function $f_{a}(s)$ of Theorem 1 is of course the function $f_{a}(S, s)$ where $S=\{2,3,5,7, \ldots\}$. The most general set $S$ of the kind required is obtained by choosing an arbitrary countably infinite subset of any positive Hamel basis of the real numbers over the rationals and then taking exponentials.

It should perhaps be emphasized that $L(S)$ is dense in $\hat{P}$ for no choice of $S$. However, every $a \in l_{1}(P)$ is completely determined by the behavior of $a$ on any set $L(S)$. This is the uniqueness theorem for Dirichlet series. The situation is a little like that obtaining in the Radon measure algebra $\mathfrak{T}(R)$ of the additive group $R$ of real numbers. Here the function $\hat{\mu}(t)$, where $\hat{\mu}(t)=\int_{-\infty}^{\infty} e^{-i x \imath} d \mu(x)(-\infty<t<+\infty)$, completely determines the measure $\mu \in \mathscr{M}(R)$. Nevertheless, the mappings $\mu \rightarrow \hat{\mu}(t)$ are not dense in the maximal ideal space of $\mathfrak{T}(R)$ (see [10]). In this case, there exist measures $\mu$ such that $|\hat{\mu}(t)|$ is bounded away from zero for all real $t$ but such that $\mu^{-1}$ does not exist 
in $\mathfrak{T}(R)$. The reason for this difference between $\mathfrak{T}(R)$ and $l_{1}(P)$ is partly explained by the absence of anything like singular measures in $l_{1}(P)$.

Note. Dr. D. A. Edwards has kindly pointed out that our Theorem 1 can be deduced from a theorem of R. S. Phillips (Theorem 2.3 of [9]). An essential part of the argument is the fact that the convolution algebra of bounded Borel measures on $[0, \infty$ [ with zero singular part has no divisors of zero. This fact is replaced in our proof by Lemmas 1 and 2.

Dr. Edwards has also remarked that although the set $\left\{\chi_{s}\right\}_{\sigma \geq 0}$ is not dense in $\hat{P}$ (that is, there are open subsets of $\hat{P}$ which do not meet it), nevertheless every sub-basic open set

$$
\left\{x:\left|\hat{a}(\chi)-\hat{a}\left(\chi^{\prime}\right)\right|<\epsilon\right\}
$$

has a nonvoid intersection with $\left\{\chi_{8}\right\}_{\sigma \geq 0}$, and this is enough for the validity of the criterion of Theorem 1 .

\section{BibLIOGRAPHY}

1. Harald Bohr, Über die Bedeutung der Potenzreihen unendlich vieler Variabeln in der Theorie der Dirichletschen Reihen $\sum a_{n} / n^{s}$, Nachrichten Königl. Gesellschaft Wiss. Göttingen, Math.-Phys. Kl. (1913) pp. 441-488.

2. I. M. Gel'fand, Über absolut konvergente trigonometrische Reihen und Integrale, Rec. Math. (Mat. Sbornik) N.S. vol. 9 (51) (1941) pp. 51-66.

3. G. H. Hardy and E. M. Wright, An introduction to the theory of numbers, Oxford, Clarendon Press, 3d ed., 1954.

4. Edwin Hewitt and H.S. Zuckerman, Harmonic analysis for certain semigroups, Proc. Nat. Acad. Sci. U.S.A. vol. 42 (1956) pp. 253-255.

5. - The $l_{1}$-algebra of a commutative semigroup, Trans. Amer. Math. Soc. vol. 83 (1956) pp. 70-97.

6. Einar Hille, The inversion problem of Möbius, Duke Math. J. vol. 3 (1937) pp. 549-568.

7. Edmund Landau, Über den Wertevorrat von $\zeta(s)$ in der Halbebene $\sigma>1$, Nachr. Ges. Wiss. Göttingen, Math.-Phys. Kl. (1933) pp. 81-91.

8. Lynn H. Loomis, An introduction to abstract harmonic analysis, New York, Van Nostrand, 1953.

9. R. S. Phillips, Spectral theory for semi-groups of linear operators, Trans. Amer. Math. Soc. vol. 71 (1951) pp. 393-415.

10. Yu. A. ŠreYder, The structure of maximal ideals in rings of measures with convolution, Rec. Math. (Mat. Sbornik) N.S. vol. 27 (69) (1950) pp. 297-318. Amer. Math. Soc. Translation no. 81, 1953.

11. Norbert Wiener, The Fourier integral and certain of its applications, Cambridge University Press, 1933. Reprinted by Dover Publications, New York.

InSTITUTE For AdVANCEd Study,

UNIVERSITY OF WASHINGTON AND

QUEEN'S UNIVERSITY, BELFAST 